\title{
Aquaporin 1 is located on the intestinal basolateral membrane in Toxocara canis and might play a role in drug uptake
}

\author{
Guangxu Ma ${ }^{1,2+}$, Aiyun Jiang ${ }^{1+}$, Yongfang Luo', Yongli Luo ${ }^{1}$, Hancheng Huang ${ }^{1}$ and Rongqiong Zhou ${ }^{1 *}$
}

\begin{abstract}
Background: Aquaporins (AQPs) are a family of integral membrane channel proteins that facilitate the transport of water and other small solutes across cell membranes. AQPs appear to play crucial roles in parasite survival and represent possible drug targets for novel intervention strategy. In this work, we investigated the tissue distribution and biological roles of an aquaporin TCAQP1 in the neglected parasitic nematode Toxocara canis.

Methods: Recombinant C-terminal hydrophilic domain of AQP1 of T. canis (rTCAQP1c) and polyclonal antibody against rTCAQP1c were produced to analyse the tissue expression of native TCAQP1 in adult (female and male) worms using an immunohistochemical approach. RNA interference (RNAi), quantitative real-time PCR (qRT-PCR) and nematocidal assays were performed to investigate the functional roles of TCAQP1 in the adult stage of T. canis.

Results: Immunofluorescence analysis showed that TCAQP1 was localised predominantly in the epithelial linings of the reproductive tract and basolateral membrane of the intestine in the adult stage (female and male) of T. canis, indicating important roles in reproduction, nutrient absorption and/or osmoregulation. Treatment with silencing RNA for $24 \mathrm{~h}$ resulted in a significant reduction of Tc-aqp-1 mRNA level in adult T. canis, though no phenotypical change was observed. The efficient gene knockdown compromised the nematocidal activity of albendazole in vitro, suggesting the role of TCAQP1 in drug uptake.

Conclusions: The findings of this study provide important information about tissue expression and functional roles of TCAQP1 protein in adult T. canis. Understanding the biological functions of this protein in other developmental stages of T. canis and related parasitic nematodes would contribute to the discovery of novel diagnostic or anthelmintic targets.
\end{abstract}

Keywords: Toxocara canis, Aquaporin 1, Tissue distribution, Reproduction, Drug uptake

\section{Background}

Transmembrane transport is a fundamental process of the cellular activity. Aquaporin 1, an integral membrane channel protein facilitating the transport of water, was first identified in human erythrocytes [1,2]. Over the years, more aquaporins (AQPs) have been identified in a number of prokaryotic and eukaryotic organisms [3-8]. Apart from water transport, these proteins have been

\footnotetext{
*Correspondence: rongqiongzhou@126.com

${ }^{\dagger}$ Guangxu Ma and Aiyun Jiang contributed equally to this work ${ }^{1}$ Department of Veterinary Medicine, College of Animal Science, Southwest University, Chongqing 402460, The People's Republic of China Full list of author information is available at the end of the article
}

shown to play roles in the transportation of glycerol, gases (e.g. $\mathrm{CO}_{2}, \mathrm{NH}_{3}, \mathrm{NO}$ and $\mathrm{O}_{2}$ ), small solutes (e.g. $\mathrm{H}_{2} \mathrm{O}_{2}$ and arsenite) and/or ions (e.g. $\mathrm{K}^{+}$and $\mathrm{Cl}^{-}$) across cell membranes $[9,10]$. However, studies indicated that these AQPs appear to be structurally and functionally divergent among organisms [11-15].

In parasites, particularly protozoans and platyhelminths, AQPs have been reported to have roles in the regulation of osmotic pressure, nutrient absorption, metabolic product efflux and/or host-parasite interactions [16-19]. Specifically, some AQPs have been proposed to play roles in the drug sensitivity and resistance of parasites, such as Leishmania spp., Trypanosoma brucei and 
Schistosoma mansoni [20-23]. These findings have been raising interest in the proposition that AQPs might represent novel therapeutic targets for parasitic diseases [24-27]. However, little is known about this area in parasitic nematodes, though selected genes coding for some AQPs have been cloned and analysed [28-30]. A better understanding of this area in parasitic nematodes would contribute to the discovery of novel anthelmintic targets.

Toxocara canis, the roundworm commonly found in canids and the main causative agent for human toxocariasis, is a parasitic nematode of public health importance to be investigated [31,32]. This parasitic nematode directly transmitted to human via a faecal-oral route [accidental ingestion of its infective eggs (from contaminated water, soil or vegetables) or infective larvae (from raw/undercooked viscera of paratenic hosts)] leads to human infection [33-35]. Young children and owners of dogs are more likely to be infected due to the higher chance of contacting with infective eggs of T. canis. Infection with this parasite usually results in allergic (e.g. pruritus and asthma) and neurological disorders (e.g. epilepsy, idiopathic Parkinson's disease and dementia) as well as eye diseases [36-41]. However, there are difficulties in the diagnosis and treatment of this parasitic disease [32, 42, 43], due to limited understanding of $T$. canis at molecular level.

Recent genomic and transcriptomic studies on this parasite have indicated that $T c-a q p-1$ might play important roles in the host-parasite interactions [44-46]. In our recent work [30], we have revealed the transcription profile of $T c-a q p-1$ in the different tissues of adult T. canis. As a logical extension, in this study, we explore the tissue expression and function of TcAQP1 protein in the adult (female and male) worms of T. canis, using immunohistochemistry, RNA interference and nematocidal assays.

\section{Methods}

\section{Parasite}

Adult $T$. canis worms were collected from naturally infected dogs, which is approved by Southwest University, China, and complied with the requirements of the Ethics Procedures and Guidelines of the People's Republic of China. Worms were washed five times in phosphate-buffered saline (PBS; pH 7.4, $37^{\circ} \mathrm{C}$ ) and then cultured in RPMI 1640 at $37{ }^{\circ} \mathrm{C}, 5 \% \mathrm{CO}_{2}$. Worms for RNA extraction were snap-frozen in liquid nitrogen and stored at $-80^{\circ} \mathrm{C}$.

\section{Prokaryotic expression of recombinant C-terminal TCAQP1}

Total RNA was extracted from the adult worms of $T$. canis using Trizol reagent (Invitrogen, Carlsbad, CA, USA) and reversely transcribed into the first-strand cDNA with M-MLV Reverse Transcriptase (Takara Bio,
Shiga, Japan). Based on the coding sequence of Tc-aqp-1 (GenBank: ALU85320), the nucleotide sequence coding for the C-terminal hydrophilic domain of TcAQP1 $\left(\mathrm{His}_{283}-\mathrm{Ala}_{310}\right)$ was amplified by polymerase chain reaction (PCR) using forward primer $5^{\prime}$-CGC GGA TCC CAC CCT TCA CCA ATT TAC ATG AA-3' (BamHI restriction sites underlined) and reverse primer $5^{\prime}$-AAA TGC GGC CGC TTA AGC GAG GTC TGA ATT TTT$3^{\prime}$ (Not $\mathrm{I}$ restriction sites underlined). The PCR product was purified and inserted into pET32a vector (Takara Bio, Shiga, Japan) via BamHI and NotI restriction sites. The recombinant plasmids were amplified in Escherichia coli DH5 $\alpha$ (Takara Bio, Shiga, Japan) and confirmed by DNA sequencing. BL21(DE3) E. coli (Takara Bio, Shiga, Japan) was transformed with the recombinant plasmids for the expression of recombinant C-terminal TcAQP1. In brief, the transformed $E$. coli was cultured in LuriaBertani broth containing $100 \mathrm{mg} / \mathrm{ml} \mathrm{ampicillin} \mathrm{till}$ $\mathrm{OD}_{600}=\sim 0.6$, then induced by $1.0 \mathrm{mM}$ of isopropy1- $\beta$ D-thiogalactoside at $37^{\circ} \mathrm{C}$ for $4 \mathrm{~h}$. Sulfate-polyacrylamide gel electrophoresis (SDS-PAGE) was used to analyse the protein expression of recombinant C-terminal TcAQP1 (rTcAQP1c). Expressed rTcAQP1c peptide was purified using a $\mathrm{Ni}^{2+}$-nitrilotriacetic-acid (Ni-NTA) resin column (Sangon Biotech, Shanghai, China) and eluted using $20 \mathrm{mM}, 50 \mathrm{mM}, 100 \mathrm{mM}, 250 \mathrm{mM}$ or $500 \mathrm{mM}$ of imidazole.

\section{Preparation of polyclonal antibody against rTCAQP1c peptide}

Pre-immune serum and polyclonal antiserum against the $\mathrm{r} T c \mathrm{AQP} 1 \mathrm{c}$ were produced by a scientific service provider GL Biochem, Shanghai. Briefly, two New Zealand white rabbits were subcutaneously injected with the purified rTcAQP1c peptide $(\sim 250 \mu \mathrm{g})$, then challenged by injecting the same amount of the antigen at 14 and 21 days following the initial injection. Rabbit anti-rTcAQP1c antiserum was harvested at seven days after the last injection. The concentration and titration of the anti-rTcAQP1c antibodies were estimated by bicinchoninic acid (BCA) assay and enzyme-linked immunosorbent assay (ELISA), respectively. To test the specificity of the polyclonal antibodies, western blotting was performed using the preimmune serum (1:5000) and polyclonal anti-rTcAQP1c serum (1:5000) as well as peroxidase-conjugated goat anti-rabbit IgG secondary antibody (1:5000).

\section{Indirect-fluorescence immunohistochemistry}

Immunofluorescence analysis was performed to determine the tissue distribution of TcAQP1 in the adult stage of $T$. canis. Adult worms (female and male) were fixed in $4 \%(\mathrm{w} / \mathrm{v})$ paraformaldehyde/PBS overnight, then embedded in paraffin. Tissue sections (5 $\mathrm{mm}$ thick) prepared 
from the fixed worms were processed with xylene, ethanol, $3 \%(\mathrm{v} / \mathrm{v}) \mathrm{H}_{2} \mathrm{O}_{2} /$ PBS and $10 \mathrm{mM}$ citrate buffer $(\mathrm{pH}$ 6.0), then blocked with $1 \%(\mathrm{v} / \mathrm{v})$ bovine serum albumin (BSA)/PBS at $4{ }^{\circ} \mathrm{C}$ overnight. The blocked sections were incubated with the rabbit anti-rTcAQP1c serum (1:500 in $1 \% \mathrm{BSA}$ ) at $37^{\circ} \mathrm{C}$ for $4 \mathrm{~h}$, fluorescein isothiocyanate (FITC)-labelled goat anti-rabbit IgG secondary antibody (1:1000 in 1\%BSA) (Solarbio, Beijing, China) at $37^{\circ} \mathrm{C}$ for $1 \mathrm{~h}$, and 4-6-diamidino-2-phenylindole (DAPI) for $5 \mathrm{~min}$. Pre-immune serum (1:500 in 1\% BSA) was used as a negative control. Fluorescence signals were detected and collected using an Olympus fluorescence imaging microscope (Tokyo, Japan).

\section{RNA interference (RNAi)}

In order to test the function of $T c A Q P 1$, we conducted RNAi assay by soaking adult worms with silencing RNA targeting $T c$ - $a q p-1$. Small interfering RNA (siRNA; $19 \mathrm{nt}$ in length) targeting $T c$ - $a q p-1$ and negative control (nonsilencing) RNA were designed using BLOCK-iT ${ }^{\mathrm{TM}}$ RNAi Designer. To check the specificity of the designed silencing RNAs (5'-GCGUGUACACUAUCUCCAA-3') and non-silencing RNA (5'-UUCUUCGAACGUGUCACG $U-3^{\prime}$ ), we manually searched these sequences against the draft genome of $T$. canis (see Zhu et al. [45]). Doublestranded RNAs with dTdT overhangs were synthesised by a scientific service provider GenePharma, Shanghai. Worms were treated with the silencing or non-silencing RNA (200 nM) in RPMI- 1640 at $37^{\circ} \mathrm{C}, 5 \% \mathrm{CO}_{2}$ for $24 \mathrm{~h}$. Nuclease-free water was used as untreated/blank control. Worm motility was checked every $6 \mathrm{~h}$. The RNAi assay was conducted in triplicate, and each replicate included $\geq 10$ worms.

\section{Quantitative real-time PCR (qRT-PCR)}

After soaking for $24 \mathrm{~h}$, the efficacy of gene knockdown was determined by comparing the relative mRNA levels of $T c$-aqp-1 between the worms treated with silencing and non-silencing RNAs. qRT-PCR was performed as described previously [30] to confirm the efficiency of gene knockdown. In brief, total RNA was extracted from an individual worm $(n=3)$ and reversely transcribed. Primer set $\left(5^{\prime}\right.$-ATG CCA GTT CGA TCT CAG CC- $3^{\prime}$ and $5^{\prime}$-ACG TGA ATG AGG GGC AAC TT-3') was used to amplify Tc-aqp-1. Small subunit ribosomal RNA (18S rRNA) (5'-AAT TGT TGG TCT TCA ACG AGG A-3' and 5'-AAA GGG CAG GGA CGT AGT CA A-3') was used as the internal standard. The relative transcriptional levels of $T c$-aqp-1 in the worms treated with silencing and non-silencing RNA were calculated using the $2^{-\Delta \Delta C t}$ method, with reference to that in untreated worms. qRT-PCR was performed in triplicate. Statistical analyses (Student's t-test) were performed using Prism 7 (GraphPad, La Jolla, USA).

\section{Nematocidal assay}

To understand whether TcAQP1 plays a role in drug uptake, we tested the nematocidal activity of albendazole on the adult worms in vitro, immediately following the RNAi assay. The worms $(n=10)$ soaked with silencing, non-silencing RNA or nuclease-free water were transferred to fresh RPMI-1640 medium supplemented with or without $0.2 \mathrm{mg} / \mathrm{ml}$ of albendazole, and incubated at $37^{\circ} \mathrm{C}, 5 \% \mathrm{CO}_{2}$ for $2 \mathrm{~h}$. Worm motility was checked for $5 \mathrm{~min}$ every $30 \mathrm{~min}$, and the number of worms survived from the albendazole treatment was counted. The nematocidal assay was conducted in triplicate. Statistical analyses (Student's t-test) were performed using Prism 7 (GraphPad, La Jolla, USA).

\section{Results}

Specific polyclonal antibody against rTCAQP1c peptide

The C-terminal hydrophilic domain of TcAQP1 was expressed in BL21 (DE3) cells as a $6 \times$ His-GST fusion protein (rTcAQP1c) (Fig. 1a). The fusion protein was enriched using a Ni-NTA chromatography column and eluted with $50 \mathrm{mM}$ of imidazole, resulting in the purified rTcAQP1c peptide (Fig. 1b). Polyclonal antibody against the $\mathrm{r} T c \mathrm{AQP} 1 \mathrm{c}$ peptide (anti-rTcAQP1c) was produced, with protein concentration estimated at $0.86 \mathrm{mg} / \mathrm{ml}$ and titration at $>1: 64,000$. Western blot analysis showed a specific binding activity of the polyclonal antibody and the rTcAQP1c peptide (Fig. 1c). No obvious binding was observed between the pre-immune serum and the rTcAQP1c peptide (Fig. 1c).

\section{Tissue distribution of TCAQP1 in adult worms}

Having produced specific polyclonal antibody against $\mathrm{r} T c \mathrm{AQP} 1 \mathrm{c}$, we sought to study the tissue distribution of native TcAQP1 in the adult stage (female and male) of T. canis. Using the anti-rTcAQP1c, tissue distribution of native TcAQP1 was indicated by indirect immunofluorescence signals (Fig. 2). Specifically, predominant fluorescence signals were detected from the reproductive tract (ovary, oviduct, uterus) of female worm (Fig. 2a), and from the seminal vesical and intestine of male adult worm (Fig. 2b). Particularly, TcAQP1 was localised in the epithelial linings of reproductive organs and intestinal basolateral membrane (Fig. 2a, b). No signal was observed in the control assay using the pre-immune serum.

\section{Gene knockdown of Tc-aqp-1}

After culturing in RPMI-1640 medium for $24 \mathrm{~h}$, the treated and untreated adult worms did not show a 


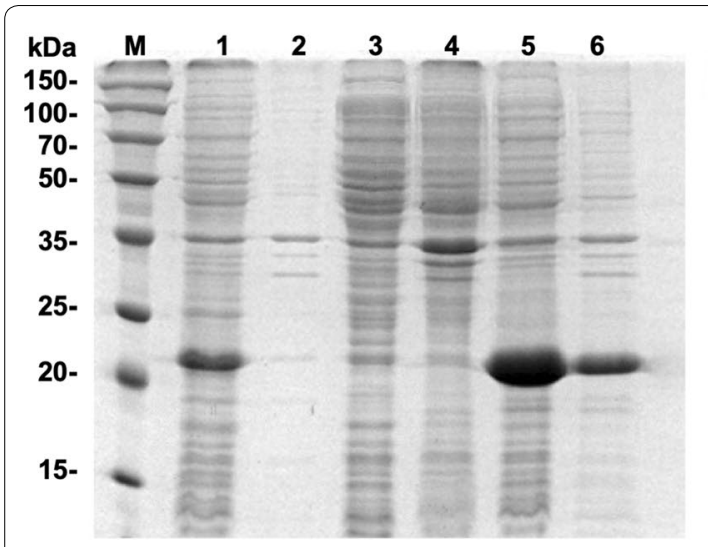

a

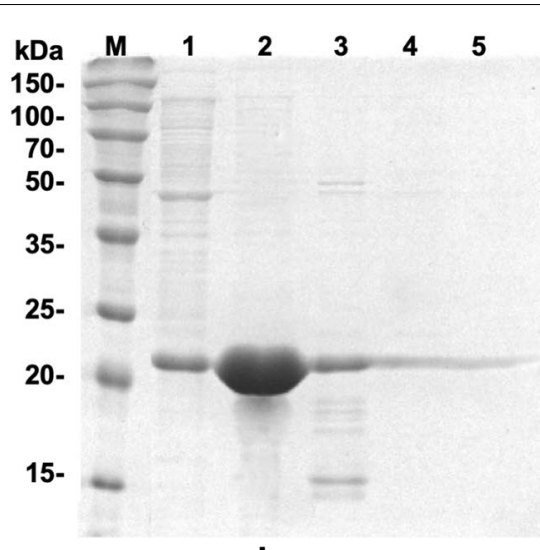

b

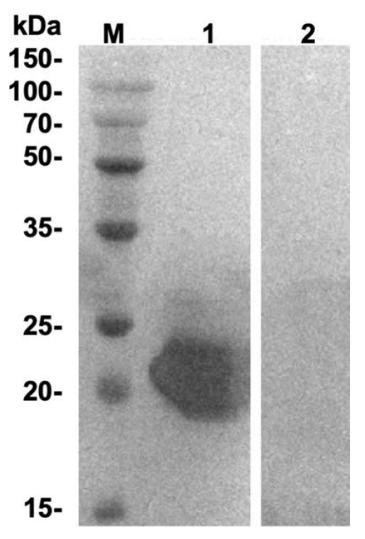

C

Fig. 1 Expression, purification and western blotting of recombinant TCAQP1c peptide. a SDS-PAGE analysis of the prokaryotic expression of recombinant TCAQP1c. Lane M: protein molecular weight maker; Lane 1: supernatant expression of induced empty pET32a; Lane 2: insoluble expression of induced empty pET32a; Lane 3: supernatant expression of non-induced recombinant pET32a plasmids; Lane 4: insoluble expression of non-induced recombinant pET32a plasmids; Lane 5: supernatant expression of induced recombinant pET32a plasmids; Lane 6: insoluble expression of induced recombinant pET32a plasmids. b Purification of recombinant TCAQP1c peptide. Lane M: protein molecular weight maker; Lanes 1-5: elution using 20 mM, 50 mM, 100 mM, 250 mM or 500 mM of imidazole, respectively. c Western blot analysis of recombinant TcAQP1c peptide. Lane M: protein molecular weight maker; Lane 1: polyclonal antibody against the recombinant TCAQP1c peptide; Lane 2: pre-immune serum

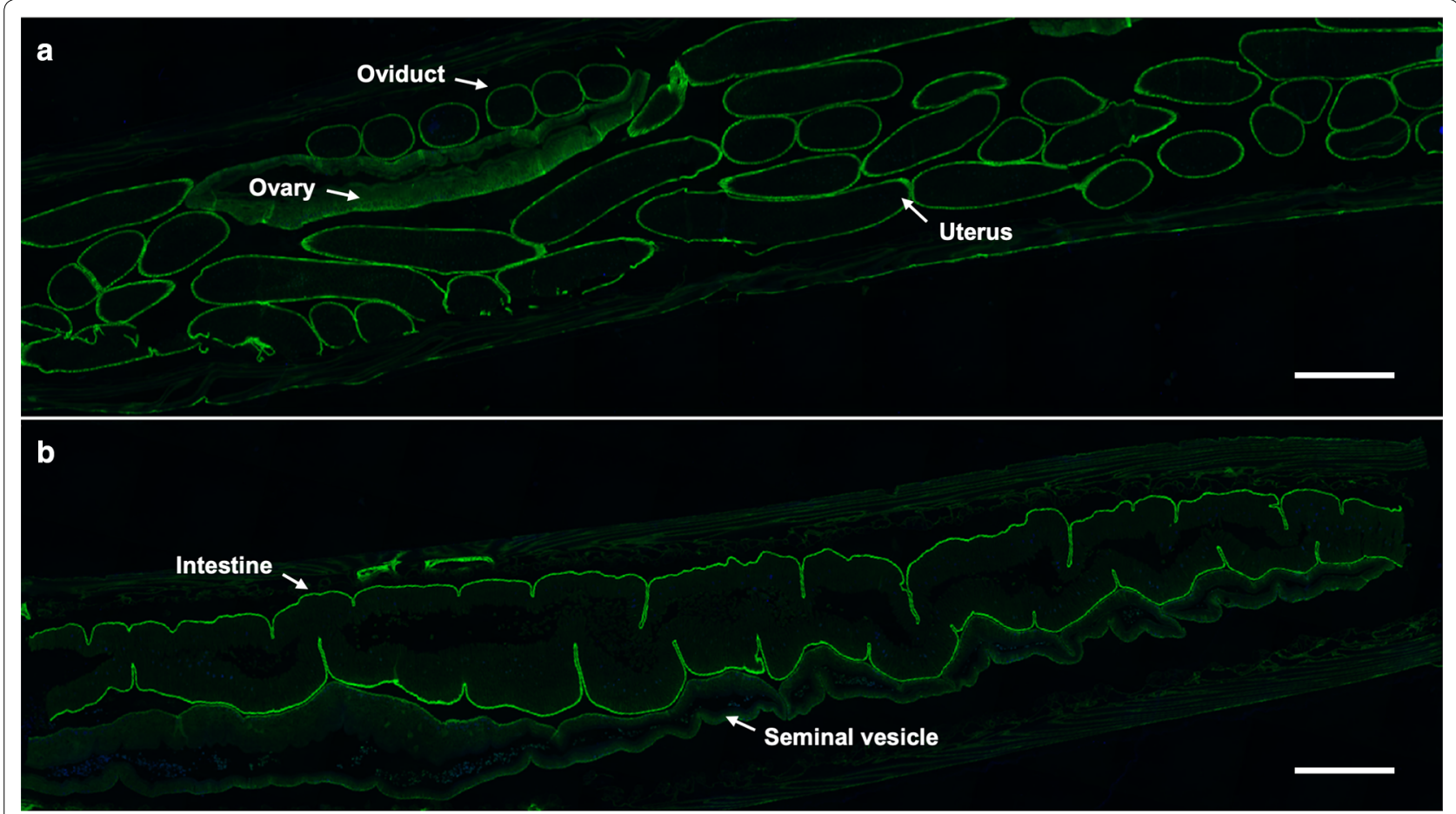

Fig. 2 Immunofluorescence microscopy of the tissue distribution of TCAQP1 in the adult stage of Toxocara canis. Tissue distribution of TcAQP1 in female (a) and male (b) adult stage of T. canis. Reproductive organs (ovary, oviduct, uterus and seminal vesicle) and intestine are indicated by white arrows. Scale-bars: $1000 \mu \mathrm{m}$

significant reduction of motility. No obvious difference in motility was seen between the worms soaked with silencing and non-silencing RNAs.
To determine whether the Tc-aqp-1 gene was efficiently silenced, we compared the mRNA level of Tc-aqp-1 between treated and untreated adult worms after soaking 
for $24 \mathrm{~h}$. We found that the siRNA (5'-GCGUGUACA CUAUCUCCAA- $3^{\prime}$ ) targeting the open reading frame of Tc-aqp-1 (G245-A263) significantly reduced the transcription of $T c-a q p-1$ in adult worms, with respect to that in untreated worms $\left(t_{(6)}=5.76, P=0.001\right.$; Fig. 3a). No significant difference was found in the mRNA levels of $T c$ aqp-1 between non-silencing RNA-treated and untreated worms $\left(t_{(6)}=0.02, P=0.098\right)$ (Fig. 3a). No obvious phenotypic change (e.g. motility) was observed in treated and untreated worms.

\section{Silenced Tc-aqp-1 compromised nematocidal activity of albendazole}

As the mRNA level of Tc-aqp-1 has been efficiently reduced in the adult worms, we sought to test whether this gene knockdown would affect the function of TcAQP1 (possibly drug transport or uptake). Treatment with $0.2 \mathrm{mg} / \mathrm{ml}$ of albendazole for $2 \mathrm{~h}$ resulted in a lethal phenotype (no motion for $5 \mathrm{~min}$ ) for $\sim 40 \%$ of the silenced worms, compared with $\sim 70 \%$ of the non-silenced worms (Fig. 3b). A significant difference $\left(t_{(6)}=5.91, P=0.098\right.$ ) was found in the nematocidal activity of albendazole between the silenced and non-silenced worms in vitro. No significant difference was found between nonsilenced and untreated worms $\left(t_{(6)}=1.00, P=0.36\right)$.

\section{Discussion}

In this study, we determined the tissue expression of TcAQP1, which is localised on the reproductive and intestinal tracts in the adult (female and male) worms of T. canis. Gene knockdown of the protein-coding gene $T c$ aqp-1 resulted in a significant reduction of mRNA level, and consequently, compromised the nematocidal activity of albendazole in vitro.

The protein expression of TcAQP1 in the epithelial linings of reproductive tract indicates biological roles in gametogenesis in adult T. canis. Similar tissue (i.e. testes and ovary) expression has been reported for AQP1 in Fasciola gigantica [14], which has been proposed to be associated with the production of seminal fluids [47]. However, in C. elegans, AQP3, rather than AQP1, has been reported to express predominantly in seminal vesicle and vas deferens [4]. The discrepancy between the free-living $C$. elegans and the parasitic $T$. canis might suggest evolutionary divergence, which clearly warrants further testing as there is a lack of information about other AQPs in the latter species. A transcriptomic study of $T c$ - $a q p-1$ or a proteomic study of TcAQP1 across the key developmental stages of $T$. canis would provide insights into the functional roles of the gene or protein in this parasite.

The predominant distribution of TcAQP1 in intestinal membrane highlights its functions in nutrient, solute transport or osmoregulation. The functional roles of AQPs in transporting water, glycerol and urea have been studied in several platyhelminths, such as F. gigantica, Opisthorchis viverrini and S. mansoni $[14,18,20]$. In these worms, AQPs have been frequently shown in the tegument cells $[14,20,48]$. Although nematodes do not have tegument, which have evolved to possess the specialised coat (cuticle), the functional roles of intestinal AQPs in nematodes should be similar to the tegument AQPs in trematodes as both of them are important organs known for nutrient absorption. This proposal can be somewhat supported by the predominant gene transcription of $T c-a q p-1$ and protein expression of TcAQP1 in the intestine of adult T. canis [30]. Interestingly, TcAQP1 was localised specifically on the intestinal basolateral membrane, which is consistent with the tissue expression of AQP1 in C. elegans [4]. This specific distribution suggests an adaptation to the chronic hypertonic stress in the intestine of the host, because it is known
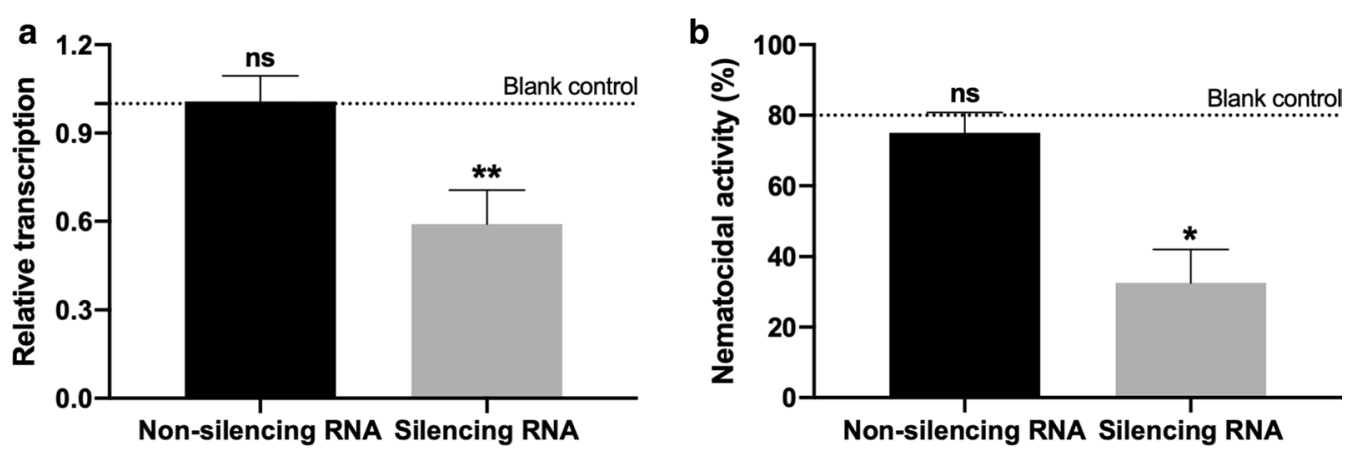

Fig. 3 Gene knockdown and nematocidal analyses. a Relative transcription of Tc-aqp-1 in adult worms soaked with non-silencing and silencing RNAs are determined with reference to blank control. $\mathbf{b}$ Nematocidal activity of albendazole on non-silenced and silenced worms are compared with respect to blank control. Error bar indicates a standard deviation (SD). Statistical significance (Student's t-test) is indicated with asterisks $\left({ }^{*} P<0.05,{ }^{* *} P<0.01\right)$ 
to transport intestinal glycerol into the pseudocoelomic cavity to maintain all non-glycerol-producing cells [49, 50]. This hypothesis should be tested by the use of genetic tools, such as RNAi and CRISPR/CAS9, which must be based on a well-established in vitro culture system.

Importantly, we silenced the gene $T c-a q p-1$ using an established soaking approach for the adult stage of $T$. canis in vitro [51]. Although the effective gene knockdown significantly resulted in reduced mRNA level of $T c$ $a q p-1$, it did not lead to a lethal or suppressed phenotype. This might be explained by the short term $(24 \mathrm{~h})$ of soaking with silencing RNA, which limited us to explore the role of $T c-a q p-1$ in reproductive activity but happened to facilitate us to test the roles of $T c-a q p-1$ in drug uptake. The roles of AQPs in drug uptake have been investigated in S. mansoni, Leishmania spp. and T. brucei [20-23], but little is known about this area in nematodes. Here, we found that the silenced (partially) Tc-aqp-1 compromised the nematocidal activity of albendazole (a broad-spectrum anthelmintic commonly used for the treatment of toxocariasis), suggesting that $T c \mathrm{AQP} 1$ might play a role in the uptake of this anthelmintic. However, this suggestion warrants further testing considering the complex pharmacokinetics of albendazole (e.g. inhibiting polymerization of $\beta$-tubulin and/or inducing degenerative alterations) [52]. A comparative analysis of the concentrations of albendazole among the tissues (intestine, reproductive organs and musculature) in the treated worms would provide a better understanding whether $T c A Q P 1$ play a role in drug uptake.

Taken together, TcAQP1 might represent a novel anthelmintic target. First, the high transcriptional level of Tc-aqp-1 in the infective larvae of $T$. canis suggests its roles in host-parasite interaction [30, 44, 45]. Second, the predominant expression of TcAQP1 in the intestine of adult $T$. canis indicates the crucial roles of this protein in parasite survival, which could be easily targeted. Although a previous immunisation with $S$. mansoni AQP1 failed to reduce worm burden and liver pathology in a murine model [53], it is still worthwhile to evaluate the potentiality of $T c \mathrm{AQP} 1$ as a novel drug target $[54,55]$, since it might play important roles in reproductive processes of T. canis. Clearly, further studies are warranted to have a better understanding of $T c-a q p-1$ or TcAQP1 in other stages of this parasitic nematodes.

\section{Conclusions}

Here, we reported for the first time the tissue distribution of TcAQP1 in the adults of T. canis, which might be associated with reproduction, nutrient absorption and/ or osmoregulation. Gene knockdown of Tc-aqp-1 compromised the nematocidal activity of albendazole in vitro, suggesting a role of TcAQP1 in drug uptake. A better understanding of this membrane channel protein would contribute to the discovery of novel intervention strategy.

\begin{abstract}
Abbreviations
Tc-aqp-1: aquaporin 1 gene of Toxocara canis; TCAQP1: aquaporin 1 protein of Toxocara canis; rTCAQP1c: recombinant the C-terminal hydrophilic domain of TCAQP1; RNAi: RNA interference; qRT-PCR: quantitative real-time polymerase chain reaction.
\end{abstract}

\section{Acknowledgements}

Not applicable.

\section{Authors' contributions}

RZ designed and supervised the study. YL, YL and AJ undertook the laboratory work. GM and RZ wrote the manuscript, with inputs from $\mathrm{HH}$. All authors read and approved the final manuscript.

\section{Funding}

This work was supported by National Natural Science Foundation of China (no. 31172313) and Fundamental Research Funds for the Central Universities (no. XDJK2017D080)

\section{Availability of data and materials}

The datasets supporting the conclusions of this article are included within the article.

Ethics approval and consent to participate

Not applicable.

Consent for publication

Not applicable.

\section{Competing interests}

The authors declare that they have no competing interests.

\section{Author details}

${ }^{1}$ Department of Veterinary Medicine, College of Animal Science, Southwest University, Chongqing 402460, The People's Republic of China. ${ }^{2}$ Faculty of Veterinary and Agricultural Sciences, The University of Melbourne, Parkville, VIC 3010, Australia.

Received: 1 March 2019 Accepted: 9 May 2019

Published online: 17 May 2019

\section{References}

1. Benga G, Popescu O, Borza V, Pop VI, Muresan A, Mocsy I, et al. Water permeability in human erythrocytes: identification of membrane proteins involved in water transport. Eur J Cell Biol. 1986;41:252-62.

2. Preston GM, Carroll TP, Guggino WB, Agre P. Appearance of water channels in Xenopus oocytes expressing red cell CHIP28 protein. Science. 1992:256:385-7.

3. Karlsson M, Fotiadis D, Sjovall S, Johansson I, Hedfalk K, Engel A, et al. Reconstitution of water channel function of an aquaporin overexpressed and purified from Pichia pastoris. FEBS Lett. 2003;537:68-72.

4. Huang CG, Lamitina T, Agre P, Strange K. Functional analysis of the aquaporin gene family in Caenorhabditis elegans. Am J Physiol Cell Physiol. 2007;292:C1867-73

5. Campbell EM, Ball A, Hoppler S, Bowman AS. Invertebrate aquaporins: a review. J Comp Physiol B. 2008;178:935-55.

6. Ishibashi K, Hara S, Kondo S. Aquaporin water channels in mammals. Clin Exp Nephrol. 2009;13:107-17.

7. Ariani A, Gepts P. Genome-wide identification and characterization of aquaporin gene family in common bean (Phaseolus vulgaris L.). Mol Genet Genomics. 2015;290:1771-85.

8. Stavang JA, Chauvigne F, Kongshaug H, Cerda J, Nilsen F, Finn RN. Phylogenomic and functional analyses of salmon lice aquaporins uncover 
the molecular diversity of the superfamily in Arthropoda. BMC Genomics. 2015;16:618.

9. Heymann JB, Engel A. Aquaporins: phylogeny, structure, and physiology of water channels. News Physiol Sci. 1999;14:187-93.

10. Verkman AS. Aquaporins. Curr Biol. 2013;23:R52-5.

11. Zardoya R. Phylogeny and evolution of the major intrinsic protein family. Biol Cell. 2005;97:397-414.

12. Gomes D, Agasse A, Thiebaud P, Delrot S, Geros H, Chaumont F. Aquaporins are multifunctional water and solute transporters highly divergent in living organisms. Biochim Biophys Acta. 2009;1788:1213-28.

13. Tornroth-Horsefield S, Hedfalk K, Fischer G, Lindkvist-Petersson K, Neutze R. Structural insights into eukaryotic aquaporin regulation. FEBS Lett. 2010:584:2580-8.

14. Geadkaew A, von Bulow J, Beitz E, Tesana S, Vichasri Grams S, Grams R. Bi-functionality of Opisthorchis viverrini aquaporins. Biochimie. 2015;108:149-59.

15. Ni ZX, Cui JM, Zhang NZ, Fu BQ. Structural and evolutionary divergence of aquaporins in parasites (Review). Mol Med Rep. 2017;15:3943-8.

16. Promeneur D, Liu Y, Maciel J, Agre P, King LS, Kumar N. Aquaglyceroporin PbAQP during intraerythrocytic development of the malaria parasite Plasmodium berghei. Proc Natl Acad Sci USA. 2007;104:2211-6.

17. Faghiri Z, Camargo SM, Huggel K, Forster IC, Ndegwa D, Verrey F, et al. The tegument of the human parasitic worm Schistosoma mansoni as an excretory organ: the surface aquaporin SmAQP is a lactate transporter. PloS One. 2010;5:e10451.

18. Thanasuwan S, Piratae S, Brindley PJ, Loukas A, Kaewkes S, Laha T. Suppression of aquaporin, a mediator of water channel control in the carcinogenic liver fluke, Opisthorchis viverrini. Parasit Vectors. 2014;7:224.

19. Cwiklinski K, Jewhurst H, McVeigh P, Barbour T, Maule AG, Tort J, et al. Infection by the helminth parasite Fasciola hepatica requires rapid regulation of metabolic, virulence, and invasive factors to adjust to its mammalian host. Mol Cell Proteomics. 2018;17:792-809.

20. Faghiri Z, Skelly PJ. The role of tegumental aquaporin from the human parasitic worm, Schistosoma mansoni, in osmoregulation and drug uptake. FASEB J. 2009:23:2780-9.

21. Mandal G, Mandal S, Sharma M, Charret KS, Papadopoulou B, Bhattacharjee $\mathrm{H}$, et al. Species-specific antimonial sensitivity in Leishmania is driven by post-transcriptional regulation of AQP1. PLoS Negl Trop Dis. 2015;9:e0003500.

22. Munday JC, Settimo L, de Koning HP. Transport proteins determine drug sensitivity and resistance in a protozoan parasite, Trypanosoma brucei. Front Pharmacol. 2015:6:32.

23. Jeacock L, Baker N, Wiedemar N, Maser P, Horn D. Aquaglyceroporin-null trypanosomes display glycerol transport defects and respiratory-inhibitor sensitivity. PLoS Pathog. 2017;13:e1006307.

24. Beitz E. Aquaporins from pathogenic protozoan parasites: structure, function and potential for chemotherapy. Biol Cell. 2005;97:373-83.

25. Fadiel A, Isokpehi RD, Stambouli N, Hamza A, Benammar-Elgaaied A, Scalise TJ. Protozoan parasite aquaporins. Expert Rev Proteomics. 2009;6:199-211.

26. Kun JF, de Carvalho EG. Novel therapeutic targets in Plasmodium falciparum: aquaglyceroporins. Expert Opin Ther Targets. 2009;13:385-94.

27. Song J, Mak E, Wu B, Beitz E. Parasite aquaporins: current developments in drug facilitation and resistance. Biochim Biophys Acta. 2014;1840:1566-73.

28. Loukas A, Hunt P, Maizels RM. Cloning and expression of an aquaporin-like gene from a parasitic nematode. Mol Biochem Parasitol. 1999;99:287-93.

29. Cui JM, Zhang NZ, Li WH, Yan HB, Fu BQ. Cloning, identification, and bioinformatics analysis of a putative aquaporin TsAQP from Trichinella spiralis. Genet Mol Res. 2015;14:12699-709.

30. Luo Y, Hu L, Ma G, Luo Y, Yin S, Xiong Y, et al. Characterization and differential expression analysis of Toxocara canis aquaporin-1 gene. Parasitol Res. 2016;115:3631-6.

31. Macpherson CN. The epidemiology and public health importance of toxocariasis: a zoonosis of global importance. Int J Parasitol. 2013:43:999-1008.

32. Ma G, Holland CV, Wang T, Hofmann A, Fan CK, Maizels RM, et al. Human toxocariasis. Lancet Infect Dis. 2018;18:e14-24.
33. Crompton DW. Ascaris and ascariasis. Adv Parasitol. 2001;48:285-375.

34. Yoshikawa M, Nishiofuku M, Moriya K, Ouji Y, Ishizaka S, Kasahara K, et al. A familial case of visceral toxocariasis due to consumption of raw bovine liver. Parasitol Int. 2008;57:525-9.

35. Strube C, Heuer L, Janecek E. Toxocara spp. infections in paratenic hosts. Vet Parasitol. 2013;193:375-89.

36. Cooper PJ. Toxocara canis infection: an important and neglected environmental risk factor for asthma? Clin Exp Allergy. 2008;38:551-3.

37. Gavignet B, Piarroux R, Aubin F, Millon L, Humbert P. Cutaneous manifestations of human toxocariasis. J Am Acad Dermatol. 2008:59:1031-42.

38. Celik T, Kaplan Y, Atas E, Oztuna D, Berilgen S. Toxocara seroprevalence in patients with idiopathic Parkinson's disease: chance association or coincidence? Biomed Res Int. 2013;2013:685196.

39. Fan CK, Holland CV, Loxton $K$, Barghouth U. Cerebral toxocariasis: silent progression to neurodegenerative disorders? Clin Microbiol Rev. 2015;28:663-86.

40. Aghaei S, Riahi SM, Rostami A, Mohammadzadeh I, Javanian M, Tohidi E, et al. Toxocara spp. infection and risk of childhood asthma: a systematic review and meta-analysis. Acta Trop. 2018;182:298-304.

41. Fialho PMM, Correa CRS, Lescano SZ. Asthma and seroconversion from Toxocara spp. infection: which comes first? Biomed Res Int. 2018;2018:4280792.

42. Othman AA. Therapeutic battle against larval toxocariasis: are we still far behind? Acta Trop. 2012;124:171-8.

43. Poulsen CS, Skov S, Yoshida A, Skallerup P, Maruyama H, Thamsborg SM, et al. Differential serodiagnostics of Toxocara canis and Toxocara cati -is it possible? Parasite Immunol. 2015;37:204-7.

44. Maizels RM. Toxocara canis: molecular basis of immune recognition and evasion. Vet Parasitol. 2013;193:365-74.

45. Zhu XQ, Korhonen PK, Cai H, Young ND, Nejsum P, von Samson-Himmelstjerna G, et al. Genetic blueprint of the zoonotic pathogen Toxocara canis. Nat Commun. 2015;6:6145.

46. Gasser RB, Korhonen PK, Zhu XQ, Young ND. Harnessing the Toxocara genome to underpin toxocariasis research and new interventions. Adv Parasitol. 2016:91:87-110.

47. Da Silva N, Silberstein C, Beaulieu V, Pietrement C, Van Hoek AN, Brown $D$, et al. Postnatal expression of aquaporins in epithelial cells of the rat epididymis. Biol Reprod. 2006;74:427-38.

48. Castro-Borges W, Simpson DM, Dowle A, Curwen RS, Thomas-Oates J, Beynon RJ, et al. Abundance of tegument surface proteins in the human blood fluke Schistosoma mansoni determined by QconCAT proteomics. J Proteomics. 2011:74:1519-33.

49. Lamitina ST, Morrison R, Moeckel GW, Strange K. Adaptation of the nematode Caenorhabditis elegans to extreme osmotic stress. Am J Physiol Cell Physiol. 2004;286:C785-91.

50. Lamitina T, Huang CG, Strange K. Genome-wide RNAi screening identifies protein damage as a regulator of osmoprotective gene expression. Proc Natl Acad Sci USA. 2006;103:12173-8.

51. Ma G, Zhou R, Song Z, Zhu H, Zhou Z, Zeng Y. Molecular mechanism of serine/threonine protein phosphatase 1 (PP1 calpha-PP1r7) in spermatogenesis of Toxocara canis. Acta Trop. 2015;149:148-54.

52. Molina AJ, Merino G, Prieto JG, Real R, Mendoza G, Alvarez Al. Absorption and metabolism of albendazole after intestinal ischemia/reperfusion. Eur J Pharm Sci. 2007;31:16-24.

53. Figueiredo BC, De Assis NR, De Morais SB, Martins VP, Ricci ND, Bicalho $\mathrm{RM}$, et al. Immunological characterization of a chimeric form of Schistosoma mansoni aquaporin in the murine model. Parasitology. 2014;141:1277-88.

54. To J, Yeo CY, Soon CH, Torres J. A generic high-throughput assay to detect aquaporin functional mutants: potential application to discovery of aquaporin inhibitors. Biochim Biophys Acta. 2015;1850:1869-76.

55. Verkman AS, Anderson MO, Papadopoulos MC. Aquaporins: important but elusive drug targets. Nat Rev Drug Discov. 2014;13:259-77.

\section{Publisher's Note}

Springer Nature remains neutral with regard to jurisdictional claims in published maps and institutional affiliations. 